\title{
Time-Domain Homogenization of Litz-Wire Bundles in 2-D FE Calculations
}

\begin{abstract}
This work deals with a time-domain homogenization technique for litz-wire bundles in finite element (FE) models. An elementary FE model is used to determine dimensionless frequency- and time-domain coefficients regarding the skin and proximity effect in litz-wire bundles. These coefficients significantly depend on the fill factor of litz-wire bundles, not the type of packing inside. With these coefficients, litz-wire bundles become homogeneous conductors which are easy to integrate into a FE model of a complete device. The method is successfully applied to a litz-wire transformer. The results agree well with the accurate reference model which all conductors in litz-wire bundles are finely discretized.
\end{abstract}

\section{Introduction}

Nowadays power converters are able to operate at higher frequencies thanks to the advent of wide-bandgap semiconductor devices. Accordingly, litz wire has become the more important element because it allows reducing the skin-effect losses at high frequencies thanks to the small strands connected in parallel as a bundle instead of using one big strand as shown in Fig. 1(b). The applications range from small switched mode power supplies to high-power solid-state transformers.

Some analytical formulas have been published in [1] to calculate losses in litz-wire, but they are not always correct in some practical cases [2]. On the other hand, FE analysis allows for higher accuracy as well as for more modeling flexibilities (e.g. including the creepage distance between each winding). However, to capture the skin and proximity effect, as a rule of thumb, the characteristic length of the discretization of the conductors should be at least three times smaller than the skin depth. This amounts to a huge number of unknowns and prohibitive computational cost and it is thus not suitable for the design process, particularly when the number of strands drastically increases in high-power applications. Thanks to the homogenization method, the litz-wire bundles are replaced by the bundles of homogeneous material of which the current density $j$ is uniform over the cross section as depicted in Fig. 1(c).

The objective of this work is to adapt and apply the homogenization technique for time-domain 2-D FE calculation in [3], which has proved successful for multi-turn-winding inductors [3],[4], to litz-wire transformers applications. Although there have been several authors publishing similar works on this topic, in [2],[5],[6], they are limited only to frequencydomain. Moreover, they focus particularly on either square or hexagonally packed conductors. The method proposed in [2] is neither implemented explicitly in terms of the fill factor of the litz-wire bundle, nor includes the skin-effect impedance. In this work, these two types of regular packings are considered and compared, and the homogenization approach is extended to time-domain simulation. This is particularly useful for power electronics applications as they are characterized by non-sinusoidal waveforms and possibly core saturation.

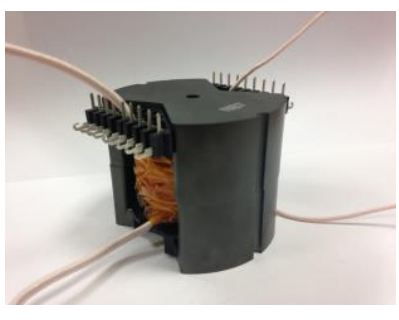

(a)

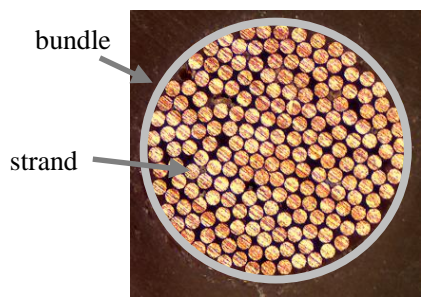

(b)

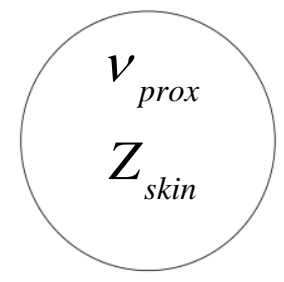

(c)

Fig. 1: (a) Transformer with litz-wire windings (b) Micrograph of cross-section of a real 0.1-mm-diameter 200-strand litz-wire bundle with $\lambda=0.4$. (c) Homogenized litz-wire bundle (with complex, frequency-dependent, fill-factor-dependent reluctivity and impedance).

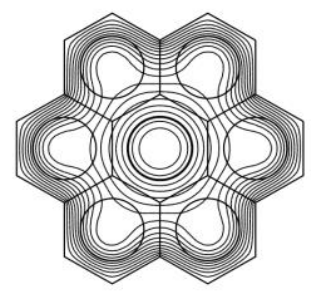

(a)

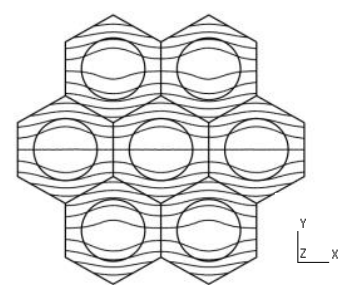

Fig. 2: Representative FE model of strand conductors of $\lambda=0.4$ at $X=2$. (a) Hexagonal packing with skin-effect flux lines (left) and proximity-effect flux lines (right). (b) Square packing with skin-effect flux lines (left) and proximity-effect flux lines (right).

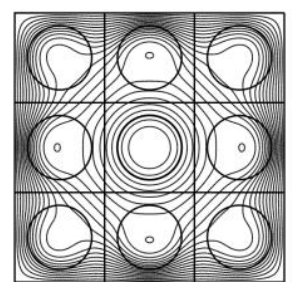

(b)

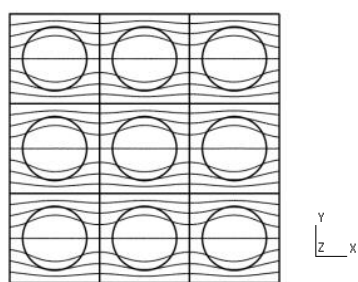




\section{Frequency-domain characterization of litz-wire bundles}

A complete eddy-current effect characterization of the strand conductors and their packing inside the litz-wire bundle can be carried out by means of a representative 2-D FE model consisting of a central cell and one or more layers of cells around it [7]. Each elementary cell comprises the cross-section of one conductor, where the skin and proximity effects occur, and the surrounding insulation. One can choose the arrangement of these cells depending on the type of packing. In general, there are two types of regular packing of round conductors in a winding: square and hexagonal. It can be observed in Fig. 1(b) that the configuration of the strands inside the bundle is not well organized, but some parts can be noticed as a hexagonal packing (mostly in the dense area). It can probably be said that the practical litz wire is a combination of these two ideal packing patterns [2]. Initially, in this section, we consider both of the square and hexagonally packed strands as the representative model as shown in Fig. 2. The skin depth at frequency $f$ or pulsation $\omega=2 \pi f$ is given by $\delta=\sqrt{2 /\left(\sigma \omega \mu_{0}\right)}$, where $\sigma$ is the conductivity of the conductors and $\mu_{0}=4 \pi 10^{-7} \mathrm{H} / \mathrm{m}$ their permeability (or reluctivity $\left.v_{0}=1 / \mu_{0}\right)$. We define the reduced frequency as follows:

$$
X=\frac{r_{\mathrm{s}}}{\delta}=\sqrt{f} \cdot r_{\mathrm{s}} \sqrt{\pi \sigma \mu_{0}}
$$

with $r_{\mathrm{s}}$ the nominal radius of the litz-wire strand. Another important parameter for the representative model is the fill factor $\lambda=n\left(r_{\mathrm{s}} / r_{\mathrm{b}}\right)^{2}$ where $r_{\mathrm{b}}$ is the radius of the litz-wire bundle and $n$ the number of strands in the bundle.

Frequency-domain 2-D FE calculations are carried out using the classical magnetic vector potential (MVP) formulation (Complex quantities are printed in bold, and $\mathbf{i}$ is imaginary unit). The skin and proximity effects are characterized in the form of a lumped frequency-dependent impedance $\boldsymbol{Z}_{\text {skin }}(X)$, and a frequency-dependent complex reluctivity $\boldsymbol{v}_{\text {prox }}(X)$ respectively using this representative model as depicted in Fig. 1(c). Thanks to the spatial orthogonality, the skin and proximity effect are decoupled. The skin-effect impedance is computed by imposing a unit current in each wire whereas the proximity-effect reluctivity is obtained by imposing, via the boundary conditions, a unit average flux density as depicted in Fig. 2. Subsequently the complex power absorbed by the central cell is computed from

$$
\boldsymbol{S}=P+\mathrm{i} Q=\frac{l}{2} \int_{\Omega_{\text {central }}}\left(j^{2} / \sigma+\mathbf{i} \omega b^{2} / \mu_{0}\right) d \Omega
$$

where $P$ and $Q$ are the active and reactive power respectively, $j^{2} / 2$ and $b^{2} / 2$ the r.m.s.-value squared of the current density and flux density respectively and $l$ the length along the third dimension ( $z$-axis). According to [7], the skin-effect frequency-dependent impedance can be written in terms of the dimensionless coefficients $p_{I}$ and $q_{I}$ as

$$
\boldsymbol{Z}_{\text {skin }}(X)=p_{I}(X) R_{\mathrm{dc}}+\mathbf{i} \omega q_{I}(X) \frac{\mu_{0} l}{8 \pi \lambda}=R_{\mathrm{dc}}\left(p_{I}(X)+\mathbf{i} q_{I}(X) \frac{X^{2}}{4 \lambda}\right)
$$

where $R_{\mathrm{dc}}=l /\left(\sigma \Omega_{\mathrm{c}}\right)$ is the DC resistance of the conductor and $\mu_{0} l /(8 \pi)$ the internal DC inductance of a round conductor with length $l$. Thus the coefficients $p_{I}$ and $q_{I}$ follow directly from $P$ and $Q$ values respectively. Analogously, for the proximity effect, the frequency-dependent complex reluctivity can be written in terms of the dimensionless coefficients $p_{B}$ and $q_{B}$ as

$$
\boldsymbol{v}_{\text {prox }}(X)=v_{0}\left(q_{B}(X)+\mathbf{i} p_{B}(X) \frac{1}{4} \lambda \sigma r_{\mathrm{s}}^{2} \omega\right)=v_{0}\left(q_{B}(X)+\mathbf{i} p_{B}(X) \frac{\lambda X^{2}}{2}\right)
$$

where the factor $\lambda \sigma r_{\mathrm{s}}^{2} \omega / 4$ in (4) follows from the analytical expression of low-frequency proximity losses in a round conductor. Likewise, the defined coefficients $p_{B}$ and $q_{B}$ follow directly from the corresponding $P$ and $Q$ values.

Fig. 3 shows the results of the defined coefficients versus reduced frequency for different values of fill factor $\lambda$ based on the explained procedure. The skin-effect losses and the corresponding coefficients $p_{I}$ are practically independent of the fill factor $\lambda$ and when the frequency is sufficiently low, i.e. $X \rightarrow 0$, the coefficients $p_{I}$ and $q_{B}$ tend to 1 as expected. It should be noted again that the recent works mostly focus on either square or hexagonally packed round conductors [2],[5],[6]. However, according to Fig. 3, we can observe that the difference of cell packing does not cause significant difference in effective complex reluctivity and impedance of a litz-wire bundle at each fill factor. Even though the discrepancy is observed in $q_{I}$, the reactive power corresponding to this skin-effect term is usually negligible compared to one corresponding to proximity-effect term in multi-winding applications. Hereinafter, the coefficients of the hexagonally packed conductors will be used.

\section{Time-domain extension}

\subsection{Skin effect}

Since the frequency-dependent skin-effect impedance is commonly approximated by a ladder circuit comprising a finite number of constant resistances and inductances, an approximate time-domain relation between instantaneous voltage $v(t)$ 

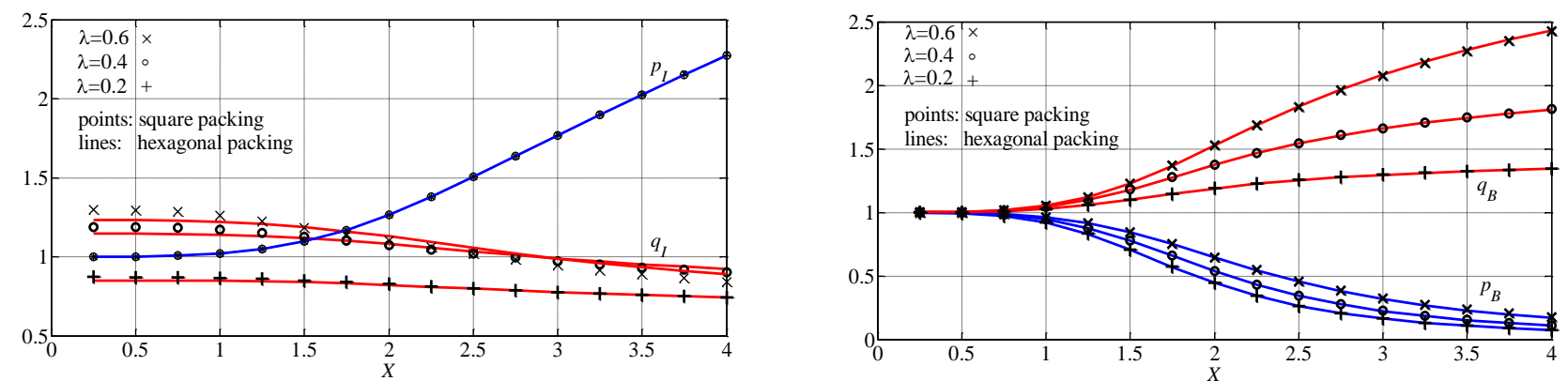

Fig. 3: Skin-effect coefficients $p_{I}$ and $q_{I}$ (left) and proximity-effect coefficients $p_{B}$ and $q_{B}$ (right) versus reduced frequency $X$ for different values of fill factor $\lambda$ for round conductors - square packing (markers) and hexagonal packing (full-lines).
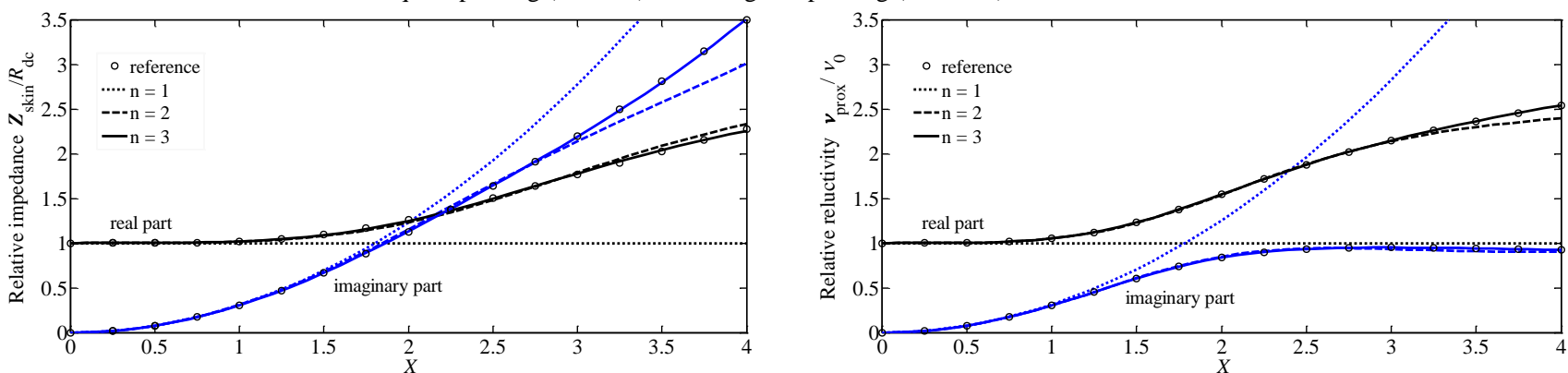

Fig. 4: Relative skin-effect impedance (left) and relative proximity-effect complex permeability (right) versus reduced frequency $X$ obtained with FE model (reference) and time-domain approximation for different values of $n=1,2,3$ at fill factor $\lambda=0.63$.

and current $i(t)$ can be formulated by considering $n-1$ auxiliary currents $[I(t)]^{T}=\left[i(t) i_{2}(t) \ldots i_{n-1}(t)\right]^{T}$ as the following system of $n$ first-order differential equations:

$$
[V(t)]=R_{\mathrm{dc}}[I(t)]+\left[L^{(n)}\right] \partial_{t}[I(t)]
$$

with $V(t)=\left[\begin{array}{llll}v(t) & 0 & \ldots & 0\end{array}\right]^{T}$ and where the square matrix $\left[L^{(n)}\right]$ has to be determined from the fitting with the obtained $\boldsymbol{Z}_{\text {skin }}(X)$ from the previous section [3]. Therefore, the system (5) is rewritten in terms of dimensionless square matrix $\left[S^{(n)}\right]$ to be in agreement with (3) as

$$
[V(t)]=R_{\mathrm{dc}}\left([I(t)]+\frac{\sigma \mu_{0} r_{\mathrm{s}}^{2}}{8 \lambda}\left[S^{(n)}\right] \partial_{t}[I(t)]\right)
$$

Converting (6) to frequency domain, we can calculate the skin-effect impedance as follows:

$$
\boldsymbol{Z}_{\mathrm{skin}}^{(n)}=\frac{\boldsymbol{v}}{\boldsymbol{i}}=R_{\mathrm{dc}}\left(\left[1^{(n)}\right]+\mathbf{i} \frac{X^{2}}{4 \lambda}\left[S^{(n)}\right]\right)_{(1,1)}^{-1}
$$

where $1^{(n)}$ is the $n \times n$ identity matrix and the subscript $(1,1)$ refers to the first diagonal element of the inverse matrix. With large enough $n$, a sufficient agreement between $\boldsymbol{Z}_{\text {skin }}(X)$ and $\boldsymbol{Z}_{\text {skin }}^{(n)}(X)$ can be achieved in the relevant frequency range $\left[0, X_{\max }\right]$. [3] and [4] show that the fitting is straightforward with a symmetric and tridiagonal matrix. The fitting with $n=1,2,3$ is demonstrated in Fig. 4 with $X_{\max }=4$ and $\lambda=0.63$. The excellent agreement can be noticed with $n=3$.

\subsection{Proximity effect}

Analogously, the dynamic relation between the instantaneous magnetic field $h(t)$ and magnetic flux density $b(t)$ can be formulated by considering $n-1$ auxiliary flux density components $[B(t)]^{T}=\left[b(t) b_{2}(t) \ldots b_{n-1}(t)\right]^{T}$. The proximity-effect counterparts of (6-7) are given by [3]

$$
\begin{gathered}
{[H(t)]=v_{0}\left([B(t)]+\frac{\lambda \sigma \mu_{0} r_{\mathrm{s}}^{2}}{4}\left[P^{(n)}\right] \partial_{t}[B(t)]\right)} \\
\boldsymbol{v}_{\text {prox }}^{(n)}(X)=\frac{\boldsymbol{h}}{\boldsymbol{b}}=v_{0}\left(\left[1^{(n)}\right]+\mathbf{i} \frac{\lambda X^{2}}{2}\left[P^{(n)}\right]\right)_{(1,1)}^{-1}
\end{gathered}
$$

with $[H(t)]^{T}=\left[\begin{array}{llll}h(t) & 0 & \ldots & 0\end{array}\right]^{T}$ and where the symmetric and tridiagonal matrix $\left[P^{(n)}\right]$ has to be fitted based on $\boldsymbol{v}_{\text {prox }}(X)$. The relative proximity-effect reluctivity $\boldsymbol{\nu}_{\mathrm{prox}} / \boldsymbol{v}_{0}$ is displayed in Fig. 4 to show the agreement between the approximation 
$\boldsymbol{v}_{\text {prox }}^{(n)}(X)$ and the reference $\boldsymbol{v}_{\text {prox }}(X)$ at different values of $n$. With $n=3$, the excellent agreement can be observed and is still valid beyond $X_{\max }=4$. It can be shown from (8) that the instantaneous magnetic energy density and joule loss density can be expressed as

$$
w_{\text {prox }}^{(n)}(t)=\frac{1}{2} v_{0}[B(t)]^{T}[B(t)] \quad \text { and } \quad p_{\text {prox }}^{(n)}(t)=\frac{\lambda \sigma r_{\mathrm{s}}^{2}}{4} \partial_{t}[B(t)]^{T}\left[P^{(n)}\right] \partial_{t}[B(t)]^{T} .
$$

\section{Application for a litz-wire transformer winding}

In Fig. 5, the 2-D FE model of a litz-wire transformer is drawn and calculated with the open-source programs Gmsh and GetDP. The transformer has translational symmetry along $z$-axis. The primary winding has 4 turns of litz-wire bundles (200-strand, $r_{\mathrm{s}}=0.2 \mathrm{~mm}, \lambda=0.43, R_{\mathrm{dc}}=0.66 \mathrm{~m} \Omega$ ). It is sandwiched by 12 turns of series-connected secondary litz-wire bundles (100-strand, $r_{\mathrm{s}}=0.2 \mathrm{~mm}, \lambda=0.63, R_{\mathrm{dc}}=3.9 \mathrm{~m} \Omega$ ). The current in each strand is assumed to be equal. Also the core is assumed to be linear and lossless (relative permeability $=1800$ ). To ensure the validity of the homogenized model, the model is used to compute the impedance of a transformer in frequency domain and joule losses in time domain compared with the results computed with the model which is finely discretized as a reference. This fine model is drawn based on the results for circle packing [8].

\subsection{Frequency-domain results}

In frequency domain, the relation between the terminal voltages $v_{1}$ and $\boldsymbol{v}_{2}$, and the terminal currents $\boldsymbol{i}_{1}$ and $\boldsymbol{i}_{2}$ for a twowinding transformer reads [9]:

$$
\boldsymbol{v}_{1}=\left(j \omega L_{1}+R_{1}\right) \boldsymbol{i}_{1}+\left(j \omega M+R_{M}\right) \boldsymbol{i}_{2} \quad \text { and } \quad \boldsymbol{v}_{2}=\left(j \omega M+R_{M}\right) \boldsymbol{i}_{1}+\left(j \omega L_{2}+R_{2}\right) \boldsymbol{i}_{2}
$$

where $L_{1}, L_{2}$ and $M$ are the self- and mutual inductances of the windings respectively, and $R_{1}, R_{2}$ and $R_{M}$ the self- and mutual resistances respectively. In FE model, these parameters can be easily calculated by proper way of excitations.

Using the $\boldsymbol{Z}_{\text {skin }}$ and $\boldsymbol{v}_{\text {prox }}$ in (3) and (4) based on the calculated coefficients, each litz-wire bundle can be homogenized and becomes a homogeneous conductor with the same diameter as the bundle in which the current density is uniform as shown in Fig. 5. In the fine model, each of the strands is finely discretized, leading to a total of 412,345 real unknowns and a computation time of $57 \mathrm{CPUs}$ (on an Intel core i7, 3.4GHz) for one time-harmonic calculation at $X=2(f=$ 2.32MHz). In the homogenized model, the discretization of the winding cells is much coarser, which results in 30 times less unknowns, viz 13,400, and a computation time of only 1.3 CPUs. In Fig. 4, similar flux patterns between fine and homogenized models can be seen. The 2-D magnetic field is present in the winding region, particularly inside the litzwire bundle, which is difficult to observe without FEA.
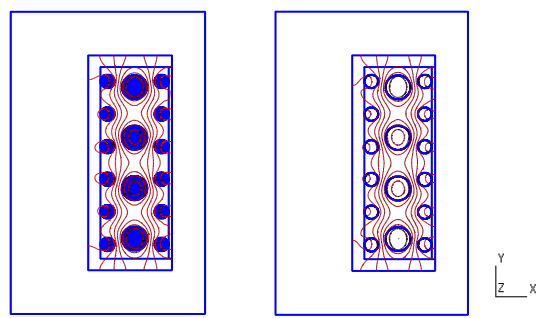

(a)

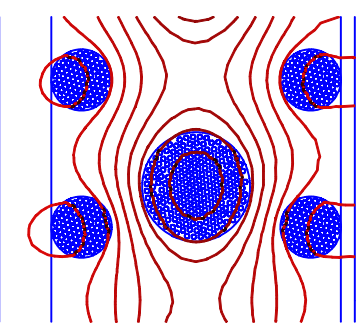

(b)

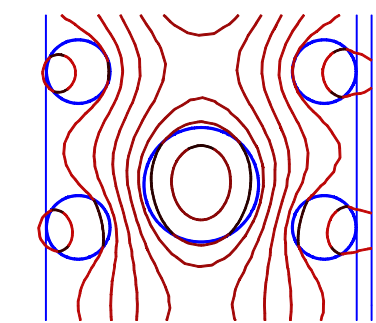

Fig. 5: 2-D model of transformer with the imposed 2.32-MHz $(X=2)$ sinusoidal primary voltage and a resistor is connected as a load at the secondary side: (a) Real part of the flux lines in the winding window (in phase with the imposed primary voltage) in fine model (left) and homogenized model (right). (b) Zoom view of the winding window (left) and homogenized model (right).

With proper excitations [9], the impedances of the transformer are calculated via the complex power in (2). However, for the homogenized model, this is done using $\boldsymbol{Z}_{\text {skin }}$ and $\boldsymbol{v}_{\text {prox }}$ rather than directly using (2). Fig. 6 shows the values of the resistance and the inductance from $X=0.2$ to $X=2$. The impedances obtained with the fine model and with the homogenized model are in good agreement. Furthermore, the increase of resistance and the slight reduction of inductance when the frequency increases can be observed. This also illustrates the significance of mutual resistance at high frequencies.

\subsection{Time-domain results}

Time-stepping simulations of the conventional DC/DC isolated full-bridge converter is carried out. The waveforms of primary and secondary currents are quasi-square waveforms of fundamental frequency $f=50 \mathrm{kHz}(X=0.3)$ which contain a lot of higher harmonic components. (The waveforms of the primary current is also affected by the trapezoidal waveform of the magnetization current.) One period is simulated with time step T/100. Fig. 7 shows how total joule losses in the 
winding vary in time. The losses is specifically high during the step changes of the current causing the rapid change of the corresponding magnetic field in (10). Still, the homogenization method produces accurate results.
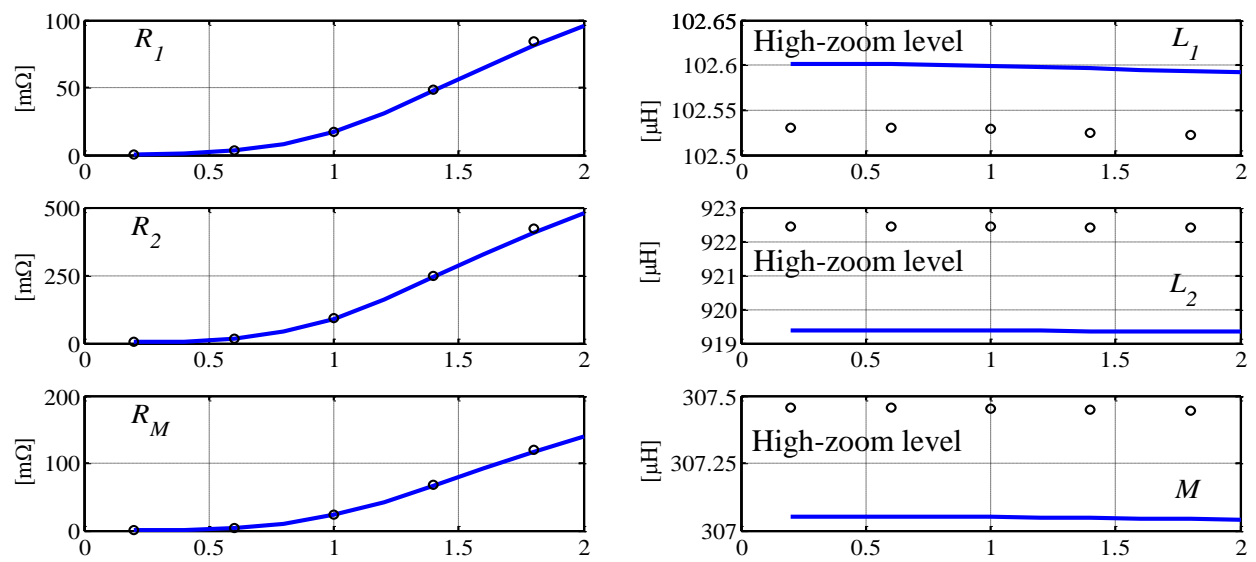

Fig. 6: Self- and mutual resistances and inductances of transformer versus reduced frequency, $X$, obtained with fine model (marker o) and homogenized model (line). The inductances are displayed in high-zoom level to show the frequency-dependent characteristic.
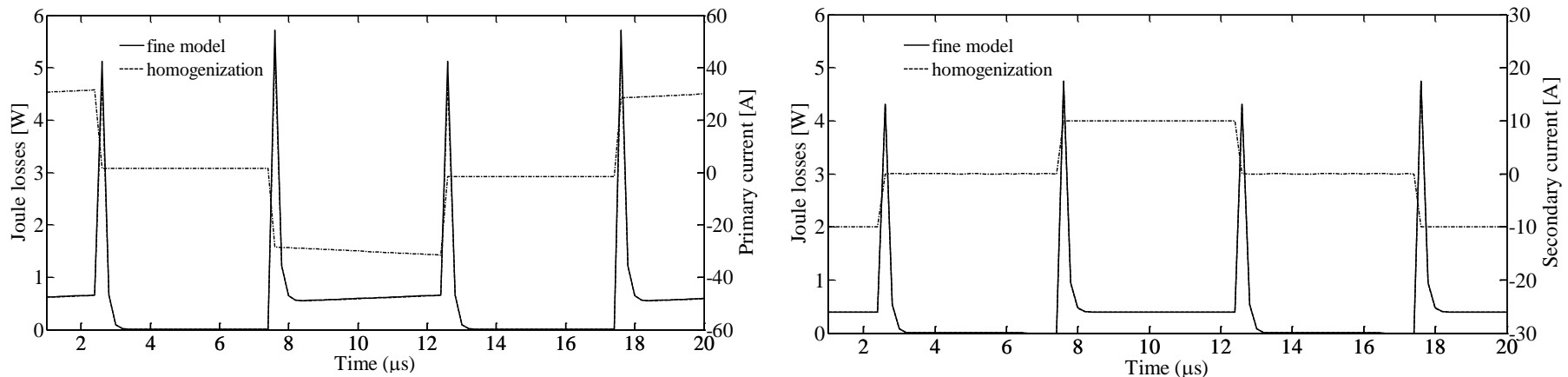

Fig. 7: Joule loess obtained with fine and homogenized model $(n=3)$ and currents versus time - primary winding (left) and secondary winding (right).

\section{Conclusion}

To homogenize a litz-wire bundle, the results has been shown that the packing of the strands inside the bundle is not significant; only fill factor is. Although the homogenized model cannot captured the detail found in the fine model, the global results of the impedances are still correct. This obviously indicates that the homogenized model can emulate the fine model of the transformer with less computational effort. This proves useful for the applications where the global results are important such as for evaluating the winding losses and for determining the inductance values to be incorporated in circuit simulators. Moreover, this method is valid in both frequency- and time-domain calculations which can solve the problem involving nonlinear magnetic core which will be elaborated more in the full paper. Also, in the full paper, the experimental result of this homogenized method based on the real transformer being used in a SiC-converter shown in Fig. 1(a) will be presented.

\section{References}

[1] J. Ferreira, "Analytical computation of ac resistance of round and rectangular litz wire windings," Proc. Inst. Elect. Eng.—Elect. Power Appl., vol. 139, no.1, pp. 21-25, Jan. 1992.

[2] X. Nan and C.R. Sullivan, "An equivalent complex permeability model for litz-wire windings," IEEE Trans. Ind. Appl., vol. 45, no. 2, pp. 854-860, 2009.

[3] J. Gyselinck, R. V. Sabariego and P. Dular, "Time-domain homogenization of windings in 2-D finite element models," IEEE Trans. Magn., vol. 43, no. 4, pp. 1297-1300, 2007.

[4] R. V. Sabariego, P. Dular and J. Gyselinck, “Time-domain homogenization of windings in 3-D finite element models," IEEE Trans. Magn., vol. 44, no. 6 , pp. 1302-1305, 2008.

[5] A. Podoltsev and B. Lebedev, "Analysis of effective resistance and eddy-current losses in multiturn winding of high-frequency magnetic components," IEEE Trans. Magn., vol. 39, no. 1, pp. 539-548, 2003.

[6] D. C. Meeker, "An improved continuum skin and proximity effect model for hexagonally packed wires," Journal of Computational and Applied Mathematics, vol. 236, no. 18, pp. 4635-4644, 2012.

[7] J. Gyselinck and P. Dular, "Frequency-domain homogenization of bundles of wires in 2D magnetodynamic FE calculations," IEEE Trans. Magn., vol. 41, no. 1, pp. 1416-1419, 2005.

[8] E. Specht, "The best known packings of equal circles in a circle," 2000-2014. [Online]. Available: http://www.packomania.com.

[9] J. H. Spreen, "Electrical terminal representation of conductor loss in transformers," IEEE Trans. Power Electron., vol. 5, no. 4 pp. 424-429, Jul. 1990. 